\title{
Balloon pulmonary angioplasty for chronic thromboembolic pulmonary hypertension
}

\author{
Marcin Kurzyna ${ }^{1}$, Szymon Darocha ${ }^{1}$, Andrzej Koteja², Radosław Pietura ${ }^{3}$, Adam Torbicki ${ }^{1}$ \\ ${ }^{1}$ Department of Pulmonary Circulation and Thromboembolic Diseases, Medical Centre of Postgraduate Education, \\ European Health Centre Otwock, Otwock, Poland \\ ${ }^{2}$ Intensive Care Unit, European Health Centre Otwock, Otwock, Poland \\ ${ }^{3}$ Department of Radiography, Medical University of Lublin, Otwock, Poland
}

Postep Kardiol Inter 2015; 11, 1 (39): 1-4

DOI: 10.5114/pwki.2015.49176

Chronic thromboembolic pulmonary hypertension (CTEPH) is a rare but potentially life-threatening disease of the pulmonary circulation [1]. The pathogenesis of $\mathrm{CTEPH}$ is not entirely clear. The most accepted scenario is that of aborted recanalisation of pulmonary arteries after a thromboembolic episode. While some post-embolic residua may persist in up to $50 \%$ of survivors of acute pulmonary embolism (aPE), only $0.5 \%$ to $2 \%$ will progress to CTEPH $[2,3]$. This is believed to occur in the presence of significant redistribution of flow to remaining unoccluded pulmonary bed with resulting elevation of intravascular pressure and shear stress. Remodelling of initially patent pulmonary arterioles leads to an increase in pulmonary vascular resistance similar to that observed in left-to-right shunting in congenital heart disease. Progressive uncoupling of pulmonary and right ventricular elastance results in a fall of pulmonary flow, left ventricular preload, systemic blood pressure, and right ventricular (RV) coronary perfusion leading to right heart failure with severe functional disability and eventually to death.

Management of CTEPH requires precise differential diagnosis and qualification for surgical treatment by an experienced multidisciplinary team. Indeed, in operable patients pulmonary endarterectomy (PEA) is highly effective in restoring functional status and improving life expectancy. A surgical technique has been optimised and implemented worldwide by a group from San Diego University of California [4]. Nevertheless, PEA performed in deep hypothermia and intermittent total cardiac arrest remains one of the most demanding cardiovascular interventions and is performed only in a limited number of highly dedicated centres. As an example, Papworth Hos- pital is the only centre performing PEA in the UK, while Marie-Lannelongue Hospital in Paris remains a referral centre for France for this type of surgery. Usually, individual cardiac surgeons are responsible for PEA in their centres, as the learning curve for this intervention has been well documented [5]. With growing experience of clinicians, radiologists, surgeons, and anaesthesiologists, an increasing proportion of patients with CTEPH may benefit from PEA despite distal, less accessible intravascular residua and/or advanced age and comorbidities. This is of paramount importance since the outcome of non-operated patients is drastically worse (Figure 1), despite identical baseline haemodynamic characteristicsand significant perioperative mortality of $2-10 \%$ in patients submitted to PEA [6]. Nevertheless, even in the leading CTEPH referral centres almost $50 \%$ patients remain on medical treatment alone, with grim perspectives regarding life quality and expectancy. Based on a large randomised trial and promising long-term effects on exercise tolerance $[7,8]$ direct guanylyl cyclase stimulator (riociguat) has been approved for treatment of inoperable CTEPH. Riociguat may protect patent pulmonary arterioles from progressive remodelling [9] but is unlikely to affect the culprit post-embolic residua. Recently, balloon pulmonary angioplasty (BPA) has emerged as a promising new interventional option in non-operable CTEPH. In 2001, Feinstein et al. from Harvard Medical School described a group of $18 \mathrm{CTEPH}$ patients treated with BPA [10]. Of these, 16 were excluded from PEA due to distal lesions, and two due to the presence of comorbidities increasing the risk associated with surgical treatment. In total, 47 procedures were performed, thus dilating or restoring

Corresponding author:

Assoc. Prof. Marcin Kurzyna MD, PhD, Department of Pulmonary Circulation and Thromboembolic Diseases, Medical Centre of Postgraduate Education, European Health Centre Otwock, 14/18 Borowa St, 05-400 Otwock, Poland, phone: +48 227103052 , fax: +48 22 710 3169 ,

e-mail: marcin.kurzyna@ecz-otwock.pl

Received: 7.01.2015, accepted: 16.01.2015. 


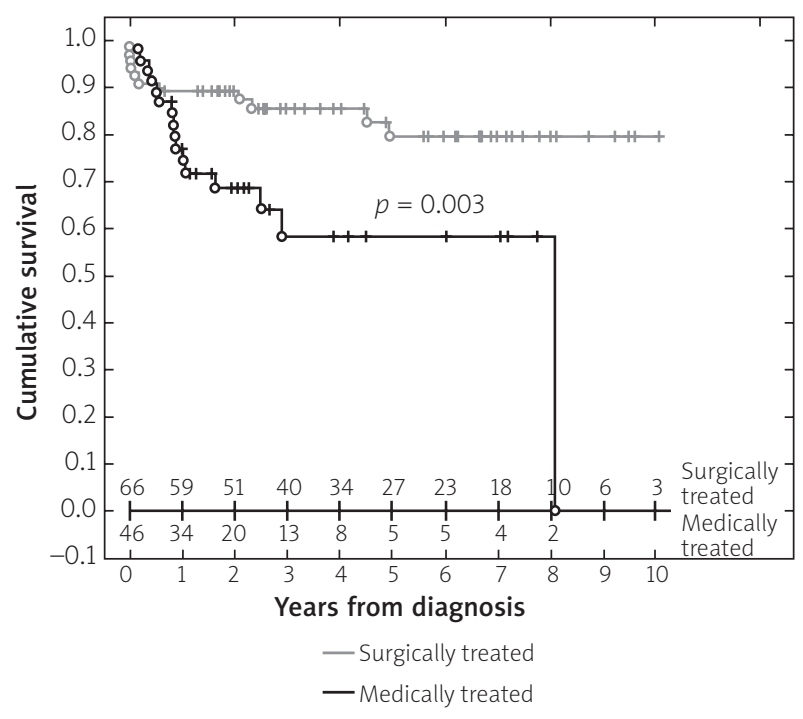

Figure 1. Survival curves for patients with CTEPH treated with PEA (shadow line) and who were treated by pharmacotherapy only (solid line) - reprinted from Wieteska et al. [6]

the patency of 107 arteries. In the periprocedural period, 1 patient died of reperfusion pulmonary oedema and right ventricular failure. In total, reperfusion oedema occurred in $23 \%$ of cases, and its presence correlated with the value of pulmonary artery pressure (PAP) prior to the procedure. Long-term follow-up (mean, 34 months) showed an increase in physical capacity, manifesting itself as an improvement in NYHA class from a mean value of 3.3 prior to the procedure to a mean value of 1.8 following the procedure.

The succeeding years saw the development of the BPA technique mainly in Japanese centres. Japanese researchers have refined the BPA technique by reducing the number of segments treated during one session [11], by using smaller balloons [12], and by wider use of intravascular imaging $[13,14]$. The experience of the Japanese centres shows that a series of BPA procedures performed in experienced centres has lead to regression of right ventricular dysfunction $[15,16]$ and are associated with an annual mortality below $5 \%$ - also in elderly patients [17]. However, it should be stressed that the population of Japanese patients with CTEPH differs from that observed in the European-American registry [18]. In a registry comprising 519 Japanese patients with CTEPH, lower median pretreatment mPAP (38 mm Hg vs. $47 \mathrm{~mm} \mathrm{Hg}$ ), more frequent use of $\mathrm{PAH}$-like therapy (52\% vs. $38 \%$ ), and a significantly lower rate of cardiac surgical treatment used (14\% vs. $57 \%$ ) was observed [19].

The only larger group of patients in Europe who underwent BPA is that described by Andreassen et al. [20]. The Norwegian team treated 20 CTEPH patients, including 16 with distal lesions, 3 with proximal lesions who had refused PEA, and 1 patient with persistent pulmonary hypertension following surgical treatment. Prior to treatment, $85 \%$ of the patients presented with NYHA class III and IV symptoms. During recruitment to a percutaneous treatment program in the same centre, 50 PEAs were performed. In total, 73 BPA procedures were carried out (mean, 3.7 BPAs/patient; range: 2-9), thus performing angioplasty of 371 vessels - 118 segmental arteries and 253 subsegmental arteries. In the periprocedural period, two patients died, and treatment-requiring reperfusion oedema occurred in 7 cases. During 3-month follow-up, an improvement in the functional class (75\% of patients in NYHA class I-II), in $\mathrm{VO}_{2 \max }$ in cardiopulmonary exercise test $(13.6 \pm 5.6$ vs. $17.0 \pm 6.5, p<0.001)$, in mean pulmonary artery pressure $(45 \pm 11 \mathrm{~mm} \mathrm{Hg}$ vs. $33 \pm 10 \mathrm{~mm} \mathrm{Hg}$, $p<0.001$ ), in pulmonary vascular resistance (8.8 \pm 4.0 Wood Units vs. $5.9 \pm 3.6$ Wood Units, $p<0.001$ ), and in NT-pro-BNP levels $(194 \pm 182 \mathrm{ng} / \mathrm{ml}$ vs. $90 \pm 119 \mathrm{ng} / \mathrm{ml}$, $p=0.007$ ) was achieved. Follow-up angiography revealed no restenosis.

In Poland, the first BPA procedure was performed in 2013 [21]. Until now, the experience of our team includes 37 BPA procedures, which consisted of angioplasty of 105 vessels in 20 patients with CTEPH. Seventeen patients were excluded from surgical treatment by an experienced PEA cardiac surgery team, and in 3 patients persistent pulmonary hypertension persisted after PEA. Eighty-two percent of patients received $\mathrm{PAH}$-like therapy - most frequently sildenafil. In the periprocedural period, two patients died of severe reperfusion oedema and severe hypoxaemia unresponsive to oxygen therapy (including mechanical ventilation). Those 2 patients were disqualified from PEA due to the presence of extensive lung cavities related to previous mycobacterial infection and due to significant comorbidities and advanced age, respectively - but not because of distal localisation of thrombi. All patients who underwent BPA because of distal lesion localisation survived.

In technical terms, BPA does not significantly differ from balloon angioplasty performed in other vessels (Figure 2). Nevertheless, the complicated anatomy of the pulmonary tree, the necessity to advance the instruments through enlarged right heart chambers, and the fact that pulmonary vessels can be easily damaged with the guide wire or balloon catheter requires specific experience. It is not recommended that BPA procedures be performed by cardiologists or interventional radiologists who have experience in other vascular regions but no experience in interventions within the pulmonary circulation. During one procedure, no more than two segmental arteries or their subsegmental equivalents should be dilated due to the risk of reperfusion oedema. Reperfusion oedema results from redistribution of blood flow to areas supplied by dilated vessels, in which vascular resistance has abruptly decreased. This may cause blood cells to migrate into the alveoli, excluding them from gas exchange. One way to prevent reperfusion oedema is to 

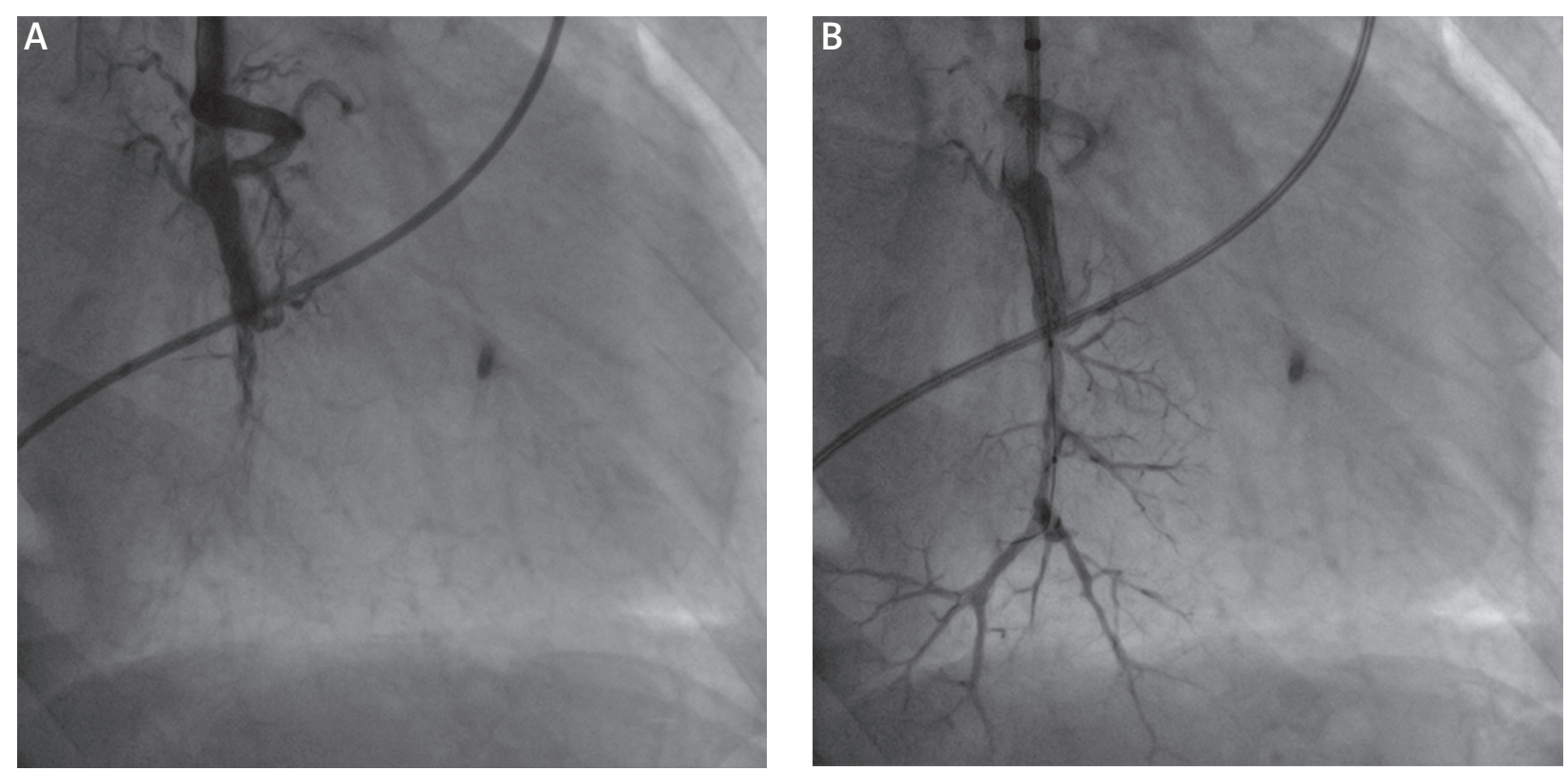

Figure 2. Balloon pulmonary angioplasty in a 67-year-old patient with persistent form of CTEPH. Left panel (A) presents the angiogram of occluded segmental pulmonary artery of left lower lobe. The BPA results in reperfusion of the vessel - right panel (B)

undersize the balloon catheter being used, on the basis of angiography, or by means of intravascular ultrasound (IVUS) or optical coherence tomography (OCT). Also, pressure distal to a residual lesion and a gradient across the lesion can be measured by means of an fractional flow reserve (FFR) probe [22]. As there is no tendency towards restenosis, it is unnecessary to use stents. The results achieved in the group of patients who have completed a series of BPA procedures are very encouraging. A reduction in mean PAP from baseline $58 \pm 6 \mathrm{~mm} \mathrm{Hg}$ to $41 \pm 9 \mathrm{~mm} \mathrm{Hg}$ and in PVR from $11.7 \pm 4.3$ Wood units to $6.6 \pm 2.2$ Wood units was achieved in our series. The haemodynamic improvement corresponds with an improvement in exercise tolerance. Prior to BPA procedures, 95\% of patients were in NYHA class III and IV, and the rate of patients in class III and IV decreased to $35 \%$ after treatment. A significant issue limiting growth in the number of such procedures performed in Poland is that BPA is not reimbursed by the National Health Fund.

\section{Future perspectives}

It cannot be denied that treatment of CTEPH is now at a crossroads [23]. Due to many years of experience and good long-term outcomes following pulmonary endarterectomy, this method will remain the first choice in patients with proximal thrombi and an acceptable perioperative risk. However, selection of patients for the procedure will not be a binary choice between a risky but potentially curative surgery and moderately or poorly effective pharmacotherapy alone. Balloon angioplasty, currently recommended in patients who do not qualify for PEA, will become an increasingly common treatment option in the future. It appears that we can witness the evolution of indications similar to that which we have been observing in transcatheter aortic valve implantation for many years now. With improvement of interventional technique, BPA will be offered to patients who could potentially be operated due to thrombus location but in whom the perioperative risk is increased. This may especially apply to patients with thrombi mainly in segmental arteries. This also opens the way to hybrid procedures not necessarily performed simultaneously - that consist of cardiac surgical removal of easily accessible lesions and interventional treatment of more distal lesions. The order of the procedures performed would remain an open question. From our preliminary experience, it may be concluded that BPA in vessels with the removed media is more difficult to perform and is associated with a higher risk of complications. As yet, there is no experience related to PEA procedures performed after interventional treatment. Another question awaiting an answer is to what extent modern PAH-like pharmacotherapy and BPA procedures are complementary, and to what extent they are alternative treatment options. The experience of Japanese centres shows that in some patients values of pulmonary artery pressure and pulmonary vascular resistance can be normalised by BPA. However, the majority of patients after BPA procedures demonstrate a significant improvement in haemodynamic parameters but still show pressure and vascular resistance values qualifying them for treatment with e.g. riociguat, according to indications described in the CHEST-1 trial [7]. 
In conclusion, BPA procedures and modern pharmacotherapy with drugs used in $\mathrm{PAH}$ so far will play an increasingly significant role in the treatment of patients with CTEPH. However, it will certainly not supersede cardiac surgery in the near future. The availability of more therapeutic options will require careful selection of patients for individual treatment methods by a team of physicians experienced in this field. Only such a multidisciplinary cardiac team including a cardiac surgeon, a cardiac anaesthesiologist, a radiologist, a cardiologist, and an interventional cardiologist experienced in procedures within the pulmonary circulation should decide about the treatment strategy of each individual patient with CTEPH, and only such teams should conduct this complex treatment.

\section{Conflict of interest}

The authors declare no conflict of interest.

\section{References}

1. Kim NH, Delcroix M, Jenkins DP, et al. Chronic thromboembolic pulmonary hypertension. J Am Coll Cardiol 2013; 62 (25 Suppl): D92-9.

2. Klok FA, van Kralingen KW, van Dijk AP, et al. Prospective cardiopulmonary screening program to detect chronic thromboembolic pulmonary hypertension in patients after acute pulmonary embolism. Haematologica 2010; 95: 970-5.

3. Konstantinides SV, Torbicki A, Agnelli G, et al. 2014 ESC guidelines on the diagnosis and management of acute pulmonary embolism. Eur Heart J 2014; 35: 3033-69.

4. Madani MM, Auger WR, Pretorius V, et al. Pulmonary endarterectomy: recent changes in a single institution's experience of more than 2,700 patients. Ann Thorac Surg 2012; 94: 97-103.

5. Mayer E, Jenkins D, Lindner J, et al. Surgical management and outcome of patients with chronic thromboembolic pulmonary hypertension: results from an international prospective registry. J Thorac Cardiovasc Surg 2011; 141: 702-10.

6. Wieteska M, Biederman A, Kurzyna M, et al. Outcome of medically versus surgically treated patients with chronic thromboembolic pulmonary hypertension. Clin Appl Thromb Hemost 2014 May 28. pii: 1076029614536604.

7. Ghofrani HA, D'Armini AM, Grimminger F, et al. Riociguat for the treatment of chronic thromboembolic pulmonary hypertension. N Engl J Med 2013; 369: 319-29.

8. Simonneau G, D'Armini AM, Ghofrani HA, et al. Riociguat for the treatment of chronic thromboembolic pulmonary hypertension: a long-term extension study (CHEST-2). Eur Respir J 2014 Nov 13. pii: erj00871-2014.

9. Ghofrani HA, Galie N, Grimminger F, et al. Riociguat for the treatment of pulmonary arterial hypertension. N Engl J Med 2013; 369: 330-40.

10. Feinstein JA, Goldhaber SZ, Lock JE, et al. Balloon pulmonary angioplasty for treatment of chronic thromboembolic pulmonary hypertension. Circulation 2001; 103: 10-3.

11. Inami T, Kataoka M, Shimura N, et al. Pulmonary edema predictive scoring index (PEPSI), a new index to predict risk of reperfusion pulmonary edema and improvement of hemodynamics in percutaneous transluminal pulmonary angioplasty. JACC Cardiovasc Interv 2013; 6: 725-36.

12. Kataoka M, Inami T, Hayashida K, et al. Percutaneous transluminal pulmonary angioplasty for the treatment of chronic thromboembolic pulmonary hypertension. Circ Cardiovasc Interv 2012; 5: 756-62.

13. Mizoguchi H, Ogawa A, Munemasa M, et al. Refined balloon pulmonary angioplasty for inoperable patients with chronic thromboembolic pulmonary hypertension. Circ Cardiovasc Interv 2012; 5: 748-55.

14. Dai Z, Fukumoto $Y$, Tatebe $S$, et al. OCT imaging for the management of pulmonary hypertension. JACC Cardiovasc Imaging 2014; 7: 843-5.

15. Fukui S, Ogo T, Morita Y, et al. Right ventricular reverse remodelling after balloon pulmonary angioplasty. Eur Respir J 2014; 43: 1394-402.

16. Tsugu T, Murata M, Kawakami T, et al. Significance of echocardiographic assessment for right ventricular function after balloon pulmonary angioplasty in patients with chronic thromboembolic induced pulmonary hypertension. Am J Cardiol 2015; 115: 256-61.

17. Yanagisawa R, Kataoka M, Inami T, et al. Safety and efficacy of percutaneous transluminal pulmonary angioplasty in elderly patients. Int J Cardiol 2014; 175: 285-9.

18. Pepke-Zaba J, Delcroix M, Lang I, et al. Chronic thromboembolic pulmonary hypertension (CTEPH): results from an international prospective registry. Circulation 2011; 124: 1973-81.

19. Tanabe N, Sugiura T, Tatsumi K. Recent progress in the diagnosis and management of chronic thromboembolic pulmonary hypertension. Respir Investig 2013; 51: 134-46.

20. Andreassen AK, Ragnarsson A, Gude E, et al. Balloon pulmonary angioplasty in patients with inoperable chronic thromboembolic pulmonary hypertension. Heart 2013; 99: 1415-20.

21. Darocha S, Kurzyna M, Pietura R, Torbicki A. Balloon pulmonary angioplasty for inoperable chronic thromboembolic pulmonary hypertension. Kardiol Pol 2013; 71: 1331.

22. Inami T, Kataoka M, Shimura N, et al. Pressure-wire-guided percutaneous transluminal pulmonary angioplasty: a breakthrough in catheter-interventional therapy for chronic thromboembolic pulmonary hypertension. JACC Cardiovasc Interv 2014; 7: 1297 306.

23. Hoeper MM. Chronic thromboembolic pulmonary hypertension at the crossroad. Eur Respir J 2014; 43: 1230-2. 